\title{
Descrição e variação sazonal na histomorfometria epididimária de Dermanura cinerea (Chiroptera: Phyllostomidae) em um fragmento de Mata Atlântica no Nordeste do Brasil
}

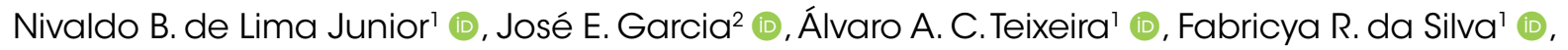 \\ Erivaldo A. Antonio' (1), Maria J. G. Arandas' (1) , Francisco C. A. de Aguiar Júnior ${ }^{2}$ (1) \\ \& Katharine R. P. dos Santos ${ }^{2}$ (1)
}

\begin{abstract}
1. Universidade Federal Rural de Pernambuco (UFRPE), Departamento de Morfologia e Fisiologia Animal (DMFA), Rua Dom Manoel de Medeiros s/n, Dois Irmãos, 52171-900 Recife, PE, Brasil (nivaldobernardo@hotmail.com)

2. Universidade Federal de Pernambuco (UFPE), Centro Acadêmico de Vitória (CAV), Núcleo de Biologia, Rua Alto do Reservatório s/n, Bela Vista, 55608-680 Vitória de Santo Antão, PE, Brasil.
\end{abstract}

RESUMO. Esse estudo objetivou avaliar sazonalmente a histomorfometria do epidídimo de Dermanura cinerea (Gervais, 1856) em um fragmento de Mata Atlântica no nordeste do Brasil. Foram utilizados 18 espécimes machos adultos capturados por redes de neblina. As coletas ocorreram mensalmente ao longo de dezoito meses, durante duas noites consecutivas e os dados meteorológicos foram fornecidos pelo Instituto Nacional de Meteorologia. Depois de eutanasiados, os espécimes tiveram os epidídimos coletados e esses órgãos foram fixados e processados. As lâminas histológicas foram coradas por Hematoxilina - Eosina e analisadas em microscopia óptica. Os parâmetros morfométricos analisados foram as áreas do túbulo, do lúmen e do epitélio das regiões do segmento inicial, cabeça, corpo e cauda do epidídimo. Os dados histomorfométricos obtidos foram submetidos às análises no teste U de Mann-Whitney. Os resultados revelaram que $D$. cinerea apresentou espermatozoides em todas as regiões do epidídimo, exceto no segmento inicial. As maiores médias das áreas tubular, do lúmen e do epitélio nas quatro regiões, foram constatadas durante os meses secos. Portanto, $D$ cinerea apresentou maior sensibilidade na região da cauda do epidídimo, ao longo dos meses com baixos índices pluviométricos. Isso indica que as condições ambientais apresentam considerável influência sobre a morfofisiologia epidídimária dessa espécie, sobretudo, em relação ao armazenamento de espermatozoides na cauda desse órgão, em área de Mata atlântica do nordeste brasileiro.

PALAVRAS-CHAVE. Epidídimo, histologia, morcegos, morfometria, sazonalidade.

ABSTRACT. Description and seasonal variation in epididymal histomorphometry of Dermanuracinerea (Chiroptera: Phyllostomidae) in a fragment of the Atlantic Forest in the Northeastern Brazil. This study aimed to evaluate seasonal patterns in the histomorphometry of the epididymis of Dermanura cinerea (Gervais, 1856) in a fragment of the Atlantic Forest in the Northeastern Brazil. Eighteen adult male specimens captured by mist net were used. The field-work occurred monthly over 18 months, during two consecutive nights. Meteorological data (precipitation) were obtained from the National Institute of Meteorology. After euthanasia, specimens had the epididymis collected, which were fixed and processed. The histological slides produced were stained by Hematoxylin - Eosin and analyzed by optical microscopy. The morphometric parameters analyzed were the tubular, lumen and epithelium areas, of the regions of the initial segment, caput, corpus and cauda of the epididymis. The histomorphometric data were submitted to the Mann-Whitney $\mathrm{U}$ test analyzes. The results showed that $D$. cinerea presented spermatozoa in all regions of the epididymis, except in the initial segment. The highest averages of the tubular, lumen and epithelial areas in the four regions were observed during the dry months. Therefore, $D$ cinerea presented greater sensibility in the region of the cauda of the epididymis, during the months with low rainfall indices. This indicates that environmental conditions have considerable influence on the epididymal morphophysiology of this species, especially in relation to the storage of sperm in the tail of this organ, in area of Atlantic forest in northeastern Brazil.

KEYWORDS. Bats, epididymis, histology, morphometry, seasonality.

A dinâmica reprodutiva das diferentes espécies de morcegos que habitam a Região Neotropical pode ocorrer de maneira sazonal ou ao longo de todo o ano. Assim, apresentam ciclos reprodutivos diferentes, os quais variam dependendo da espécie ou da área de ocorrência considerada, posto que uma mesma espécie pode ter ciclos e padrões reprodutivos divergentes em locais distintos, isto porque pode ser influenciada por fatores abióticos e pela disponibilidade de recursos alimentares (FLEMING et al., 1972; WILSON, 1973, 1979; TAdDEI, 1980; FABIÁN \& MARQUes, 1989; Crichton \& KRUTZSCH, 2000; NeUweIler, 2000; ZorTÉA, 2003).

Com a finalidade de ajustar seu organismo às condições do meio no qual estão inseridos, os morcegos desenvolveram ao longo do tempo as mais diversas estratégias reprodutivas. Dentre estas, é possível destacar o prolongamento da cauda do epidídimo, quando comparada 
às demais regiões desse órgão, o qual é responsável pelo transporte, armazenamento e maturação dos espermatozoides (LeÓN-Galván et al., 2005; ToBón et al., 2010; Beguelini et al., 2013a).

Além disso, os quirópteros podem apresentar características peculiares no processo de armazenamento dos espermatozoides durante um longo período, de forma que a viabilidade destes seja preservada. Nos morcegos neotropicais esse armazenamento na cauda do epidídimo pode ser viável por um período de 2 a 7 meses, contudo esse tempo pode variar de acordo com a espécie e hábitat considerado. Com isso, o conhecimento acerca da histofisiologia epididimária, entre as diferentes espécies de morcegos, necessita ser averiguado para melhor elucidar sobre as adaptações e variações existentes entre as espécies (Gustafson, 1979; Altringham, 1996; Hosken et al., 1998; Crichton \& Krutzsch, 2000; ENCARNAÇão et al., 2003; Rochel et al., 2007; Товón et al., 2010; BEguelini et al., 2015).

Essa relação das variações sazonais na reprodução dos morcegos ainda tem sido pouco estudada, sobretudo, quando se considera os parâmetros morfofisiológicos e morfométricos do epidídimo. Algumas investigações que abordam a morfologia, bem como os tipos celulares encontrados nas regiões desse órgão nos quirópteros, foram realizadas em algumas espécies como Corynorhinus mexicanus (G. M. Allen, 1916) (CERVANTES et al., 2008), Eumops glaucinuss (Wagner, 1843) e Molossus molossus (Pallas, 1766) (Beguelini et al., 2010), Peropteryx macrotis (Wagner, 1843) (Beguelini et al., 2012), Artibeus planirostris (Spix, 1823) (Beguelini et al., 2012, 2013b), Artibeus lituratus (Olfers, 1818) (Oliveira et al., 2013; Campolina-Silva et al., 2018), Myotis nigricans (Schinz, 1821) (BEGUELINI et al., 2012, 2013c, 2015) e Desmodus rotundus (E. Geoffroy, 1810) (CASTRO et al., 2017; SouZa et al., 2018). Entretanto, estudos com essa abordagem e com inferências acerca da influência da sazonalidade nesse órgão ainda são inexistentes no estado de Pernambuco, inclusive para Dermanura cinerea (Gervais, 1856).

Dermanura cinerea é uma espécie que era conhecida como Artibeus cinereus (Gervais, 1856) e que integra a família Phyllostomidae (REDONDO et al., 2008; SolaRI et al., 2009). Tem hábito alimentar frugívoro, atua na dispersão de sementes e encontra-se distribuída amplamente em áreas de matas ciliares primárias e fragmentos de Mata Atlântica (SCULtori et al., 2009; Reis et al., 2011). As informações sobre sua biologia reprodutiva ainda permanece pouco compreendida e embora já tenha sido relatado o padrão de poliestria bimodal (Rocha et al., 2010; LIMA JUNIOR et al., 2018a,b) em algumas localidades, as investigações referentes ao epidídimo dessa espécie são escassas.

Diante do exposto e tendo em vista que a sazonalidade pode interferir no processo reprodutivo de diferentes espécies de morcegos, o presente estudo avaliou a variação sazonal da histomorfometria epididimária de $D$. cinerea em um fragmento de Mata Atlântica situado no Litoral Sul do estado de Pernambuco, na Região Nordeste do Brasil.

\section{MATERIAL E MÉTODOS}

Área de estudo e coleta dos dados. Foram utilizados 18 espécimes machos adultos de $D$. cinerea coletados em um fragmento de Mata Atlântica. A região apresenta clima Tropical úmido com chuvas de inverno-outono e vegetação característica de Floresta Ombrófila Densa das Terras Baixas e Sistema Secundário, situado a aproximadamente $100 \mathrm{~km}$ ao sul - Sudoeste de Recife (0843'35.3'S e 3508'59.9'W) cuja área de abrangência, pertence aos municípios de Rio Formoso e Tamandaré - Estado de Pernambuco.

A captura desses animais foi realizada por meio de redes de neblina de $12 \mathrm{~m}$ de comprimento $\mathrm{x} 3 \mathrm{~m}$ de altura, de forma que foram utilizadas dez redes em cada noite de captura. As coletas ocorreram ao longo de dezoito meses (junho/2014 a novembro/2015), sendo realizadas mensalmente durante duas noites consecutivas.

Em cada noite de captura as redes eram abertas às $17 \mathrm{~h} 00 \mathrm{~min}$, permanecendo operantes até às $00 \mathrm{~h} 00 \mathrm{~min}$ e verificadas em intervalos regulares de 15 minutos. O esforço de captura foi calculado com base na metodologia de Straube \& Bianconi (2002), de modo que ao longo do período de coleta considerado obteve-se um esforço total de $90720 \mathrm{~m}^{2}$.h.

O estudo foi aprovado pelo Comitê de Ética em Experimentação Animal (CEUA) da Universidade Federal de Pernambuco (UFPE) com o número de protocolo 23076.037360/2014-92.

Dados meteorológicos. As médias referentes à temperatura $\left({ }^{\circ} \mathrm{C}\right)(\mathrm{Tab} . \mathrm{I})$ e precipitação $(\mathrm{mm})$ foram disponibilizadas pelo Instituto Nacional de Meteorologia - INMET. A partir dos dados mensais da precipitação, foi definida a mediana de valor 130,95 milímetros (mm), de modo que dois agrupamentos foram organizados compostos por nove meses cada. Os meses com índices pluviométricos maiores que a mediana foram classificados como meses chuvosos (junho, julho, agosto, setembro, outubro/2014 e março, maio, junho, julho/2015) e os meses que tiveram a pluviosidade inferior à mediana foram categorizados como secos (novembro, dezembro/2014 e janeiro, fevereiro, abril, agosto, setembro, outubro, novembro/2015) (LiMA JUNIOR et al., 2018b).

Eutanásia dos animais. Os animais utilizados nas análises histomorfométricas receberam inicialmente, anestesia na região intraperitoneal do corpo, sendo a mesma constituída pelo fármaco pentobarbital sódico em uma concentração de $40 \mathrm{mg} / \mathrm{kg}^{-1}$. Na sequência, foi aplicada uma solução saturada constituída por cloreto de potássio (Morais et al., 2013).

Coleta dos órgãos e análise histológica. Após a eutanásia, os epidídimos foram coletados juntamente com os testículos por meio de uma incisão da pele e fáscias escrotal na região inguinal, para expor o tecido subjacente até que esses órgãos fossem expostos e coletados.

Depois de removidos, os epidídimos foram fixados em formalina a $10 \%$ neutra tamponada (NBF), durante 24 horas, e conservados em álcool a $70 \%$ e glicerinado a $5 \%$. Na 
Tab. I. Médias mensais referentes à temperatura $\left({ }^{\circ} \mathrm{C}\right)$ de acordo com o Instituto Nacional de Meteorologia (INMET)/Seção de Observação e Meteorologia Aplicada (SEOMA) - Pernambuco, Mata Sul - 2014 e 2015.

\begin{tabular}{clll}
\hline Mês & Ano & Temperatura $\left({ }^{\circ} \mathrm{C}\right)$ \\
\hline Junho & 2014 & 24,9 \\
Julho & 2014 & 24,4 \\
Agosto & 2014 & 24,0 & 24,8 \\
Setembro & 2014 & 25,7 \\
Outubro & 2014 & 26,3 \\
Novembro & 2014 & 26,7 \\
Dezembro & 2014 & 26,9 \\
Janeiro & 2015 & 27,4 \\
Fevereiro & 2015 & 26,8 \\
Março & 2015 & 27,2 \\
Abril & 2015 & 26,4 \\
Maio & 2015 & 25,0 \\
Junho & 2015 & 24,3 \\
Julho & 2015 & 24,6 \\
Agosto & 2015 & 25,7 \\
Setembro & 2015 & 26,4 \\
Outubro & 2015 & 27,0 \\
Novembro & 2015 & \\
\hline
\end{tabular}

sequência, foram processados seguindo a técnica histológica de rotina (BEHMER et al., 1976; RIEDER \& SCHMIDT, 1987).

Os órgãos foram impregnados em Paraplast, produzidos os blocos e cortados no micrótomo em espessura de $4 \mu \mathrm{m}$. As lâminas histológicas foram coradas por Hematoxilina - Eosina (H.E.) e submetidas às análises em microscopia óptica para observar os tipos celulares e demais estruturas no epidídimo.

Análises morfométricas. As lâminas histológicas foram fotografadas em aumento total de $100 \mathrm{X}$, por meio do software Motic Images Plus 2.0. A mensuração da área tubular (AT), área do lúmen (AL) e área do epitélio (AE) de cada região do epidídimo foi realizada por meio do programa ImageJ. Para isso, foram utilizadas 10 fotomicrografias e considerados 15 túbulos por região de cada animal. Os resultados obtidos para $\mathrm{AT}, \mathrm{AL}$ e $\mathrm{AE}$ foram expressos em micrômetros quadrados $\left(\mu \mathrm{m}^{2}\right)$.

Análise estatística. Os resultados obtidos na morfometria, para cada uma das regiões do epidídimo, foram submetidos ao programa SPSS (Statical Package of the Social Sciences, SPSS inc. Chicago, EUA) versão 15.0. Primeiramente, foi realizado o teste de Kolmogorov-Smirnov, para averiguar se a distribuição dos dados era normal. Em seguida, foi utilizado o teste $U$ de Mann-Whitney para avaliar os parâmetros morfométricos do epidídimo, de forma que foi considerada a significância de $5 \%(\mathrm{p}<0,05)$ nas análises realizadas.

\section{RESULTADOS E DISCUSSÃO}

Os resultados das análises histológicas indicaram que o epidídimo de Dermanura cinerea apresenta quatro regiões: segmento inicial (SI), cabeça (CA), corpo (CP) e cauda (CAU) (Figs 1-4). O SI está localizado entre a convergência tubular da rede testicular e a CA do epidídimo. O CP consiste em uma região intermediária entre a cabeça e a região terminal (cauda). Esta última região mantém comunicação direta com o ducto deferente.

O epidídimo é constituído por epitélio, lúmen e túnica própria. Circundando esse órgão, encontra-se o intertúbulo que é composto por tecido conjuntivo contendo vasos sanguíneos e células musculares lisas. Esse tecido continua-se com a túnica própria, que envolve os túbulos epididimários, sobre a qual repousa o epitélio pseudoestratificado com estereocílios que possui diferentes tipos de células (basal, principal, halo, clara, estreita e apical). O SI apresentou um epitélio irregular e mais baixo, enquanto que a CA exibiu um epitélio mais alto. O CP apresentou-se como a mais alongada e a CAU como a de maior espaço luminal (PASTOR-SOLER et al., 2010; RoBAIRE \& Hinton, 2015).

Os espécimes apresentaram espermatozoides nas regiões (CA, CP e CAU), exceto no SI do epidídimo. À medida que se direcionava para a região terminal do órgão, observou-se aumento na concentração de espermatozoides, sobretudo, na CAU. De acordo com BEGUELINI et al. (2013a), a região da cauda do epidídimo é o local onde há maior concentração de espermatozoides, independentemente da posição testicular (ToBón et al., 2010; LIMA JUNIOR et al., 2014). Este fato pode estar associado à funcionalidade e atuação dessa região no processo de metabolismo do esperma, bem como na maturação dos espermatozoides até que sejam liberados durante a ejaculação, como ocorre na cauda do epidídimo dos demais mamíferos (BUDRAS \& SAUER, 1975; Artoni et al., 1999; CoOper, 1999). 
Nas análises morfométricas das áreas tubular (AT), do lúmen $(\mathrm{AL})$ e do epitélio $(\mathrm{AE})$ de cada região ficou constatado que para todos esses três parâmetros avaliados, $D$. cinerea apresentou as maiores médias ao longo de meses secos e esses resultados foram significativos para todas as regiões
(SI, CA, CP e CAU) do epidídimo. Além disso, de maneira geral, nota-se que a AT, AL e AE aumentaram entre as regiões à medida que se direciona para a porção da cauda. Assim, o SI apresentou as menores médias e a CAU as maiores quando comparadas às demais regiões desse órgão (Figs 5-7; Tab. II).

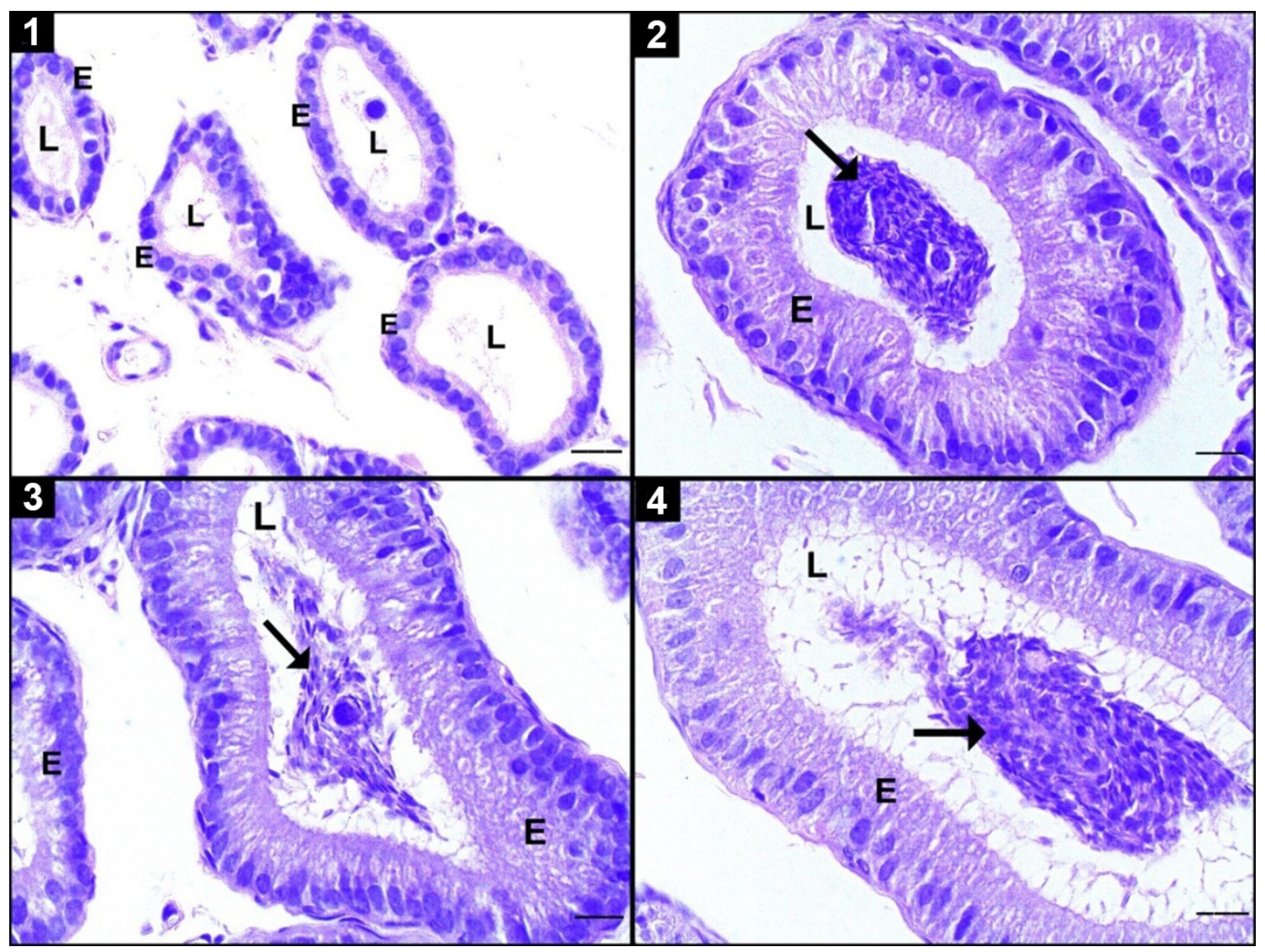

Figs.1-4. Fotomicrografias das quatro regiões do epidídimo de Dermanura cinerea (Gervais, 1856): 1, segmento inicial; 2, cabeça; 3, corpo; 4, cauda (E, epitélio; L, lúmen; setas, espermatozoides). Coloração: H.E. Aumento total: 400X. Barra de escala: $20 \mu \mathrm{m}$.

Tab. II. Média e desvio padrão da área do túbulo (AT), área do lúmen (AL) e área do epitélio (AE) das regiões do epidídimo de Dermanura cinerea (Gervais, 1856) coletados durante os meses chuvosos e secos em área de Mata Atlântica no Litoral Sul de Pernambuco. *, Parâmetros mensurados em micrômetros quadrados $\left(\mu \mathrm{m}^{2}\right)$.

\begin{tabular}{lllll}
\hline & & $\begin{array}{l}\text { Meses chuvosos } \\
(\mathrm{n}=11)\end{array}$ & $\begin{array}{l}\text { Meses secos } \\
(\mathrm{n}=07)\end{array}$ & Valor de $\mathrm{p}$ \\
\hline Regiões do epidídimo & Parâmetros* & Média e desvio padrão & Média e desvio padrão & $<0,0001$ \\
\hline \multirow{3}{*}{ SI } & AT & $4098,68 \pm 3175,99$ & $8611,63 \pm 4761,72$ & $<0,0001$ \\
& AL & $1208,42 \pm 1192,56$ & $2305,01 \pm 1633,99$ & $<0,0001$ \\
& AE & $2890,26 \pm 2252,07$ & $6306,62 \pm 3631,40$ & $<0,0001$ \\
\hline CA & AT & $7150,68 \pm 5399,61$ & $12109,25 \pm 7384,70$ & $<0,0001$ \\
& AL & $2207,38 \pm 2336,39$ & $3441,06 \pm 3056,48$ & $<0,0001$ \\
CP & AE & $5017,26 \pm 3326,39$ & $8668,19 \pm 5068,81$ & $<0,0001$ \\
\hline & AT & $6693,87 \pm 3719,11$ & $11779,22 \pm 5922,75$ & $<0,0001$ \\
CAU & AL & $2046,77 \pm 1176,15$ & $3593,94 \pm 3206,08$ & $<0,0001$ \\
& AE & $4646,09 \pm 2233,47$ & $8185,28 \pm 3957,74$ & $<0,0001$ \\
\hline AT & $14138,17 \pm 13255,17$ & $22186,53 \pm 15878,33$ & $<0,0001$ \\
& AL & $7735,22 \pm 9499,74$ & $12708,20 \pm 10845,12$ & $<0,0001$ \\
\end{tabular}



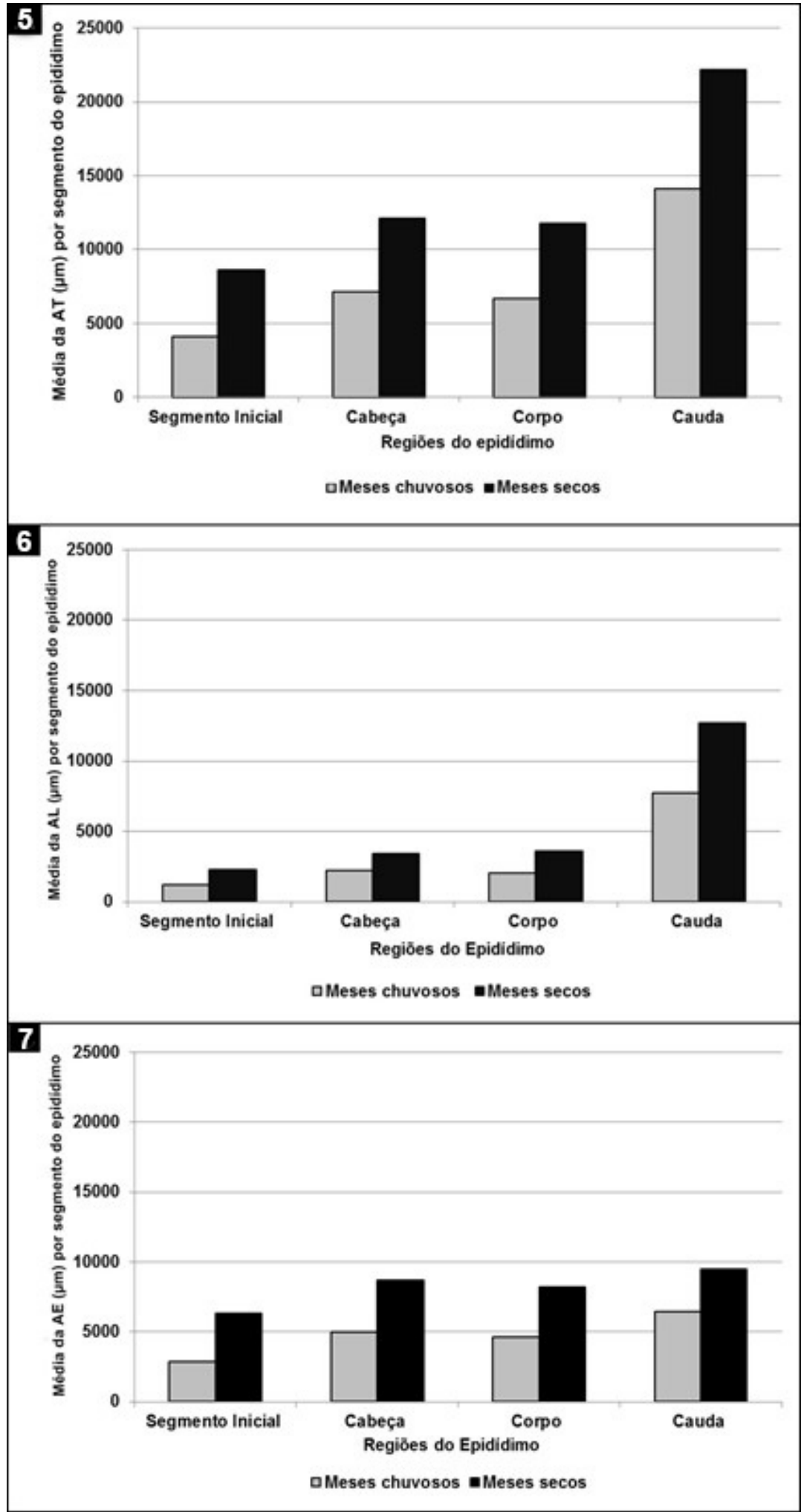

Figs 5-7. Gráficos referentes às médias da área do túbulo - AT (Fig. 5), área do lúmen - AL (Fig. 6) e área do epitélio - AE (Fig. 7) das quatro regiões (segmento inicial, cabeça, corpo e cauda) do epidídimo de Dermanura cinerea (Gervais, 1856), coletados em meses chuvosos e secos, em área de Mata Atlântica no litoral Sul de Pernambuco, Brasil.

As maiores médias apresentadas na cauda do epidídimo reforçam as registradas para outras espécies. Assim, dentre as regiões do epidídimo, a mais sensível às mudanças ocasionadas pela sazonalidade consistiu na região da cauda, indicada pelo aumento das áreas do túbulo, lúmen e epitélio. Essas variações sazonais possivelmente têm relações com as alterações nos níveis séricos de testosterona ao longo do ciclo reprodutivo anual, visto que esse hormônio, 
associado aos diferentes tipos celulares existentes no epitélio desse órgão, tem fundamental importância no processo de maturação e viabilidade dos espermatozoides ao longo de todas as regiões do epidídimo e, sobretudo, na região caudal na qual essas células ficam armazenadas até serem expelidas por meio da ejaculação (CALvo et al., 1997, 1999; Aguilera-Merlo et al., 2005; Beguelini et al., 2013c, 2015; CASTRO et al., 2017).

O armazenamento, bem como o tempo de duração dos espermatozoides na cauda do epidídimo, pode variar, pois após o fim da espermatogênese, ainda é possível que a estocagem permaneça. Essa funcionalidade possibilita que os machos sincronizem sua atividade reprodutiva com a chegada de fêmeas receptivas após o término da espermatogênese, de forma que o acasalamento e a fecundação possam acontecer de maneira eficaz. Assim, essa região do epidídimo tem importância histofisiológica no armazenamento e manutenção dos espermatozoides viáveis por um longo período de tempo (COOPER, 1999; Tobón et al., 2010; Lima Junior et al., 2018a,b).

A elevação das médias constatadas nos meses secos, sobretudo na região da cauda, está relacionada com as alterações fisiológicas das regiões do epidídimo de $D$. cinerea, influenciadas pelas condições ambientais. A maior área do lúmen indica um aumento e, consequentemente, uma maior quantidade de espermatozoides que poderão estar distribuídos ao longo desse espaço. Por outro lado, quando ocorre uma diminuição na área luminal dessa região, possivelmente há também uma diminuição na quantidade de espermatozoides. Desse modo, $D$. cinerea apresentou o ambiente luminal do epidídimo mais propício para motilidade dessas células reprodutivas masculina nos meses secos, pois nestes, tal órgão passa por mudanças morfofisiológicas importantes para promover maior desempenho na atividade reprodutiva dos machos e também para sincronizar com o período fértil das fêmeas, o que possibilita a ocorrência do acasalamento em meses secos, já que durante estes as fêmeas encontram-se receptivas para entrar em período gestacional e parir em meses chuvosos, os quais apresentam recursos favoráveis para manter a prole (Lima Junior et al., 2018a,b). De forma análoga, as maiores médias da área do túbulo e do epitélio expressam uma preparação tecidual para a produção dos diferentes tipos celulares (basal, principal, halo, clara, estreita e apical) que atuarão com funções específicas de acordo com a região do epidídimo na qual se encontram, podendo, por exemplo, atuar na absorção, secreção, síntese e metabolismo durante os meses com baixos índices pluviométricos (HINTON, 1990; Turner, 1991; Serre \& Robaire, 1998; Beguelini et al., 2010, CASTro et al., 2017).

Os resultados apresentados revelam que $D$. cinerea demonstra maior sensibilidade às variações sazonais na região da cauda do epidídimo. Ademais, essa espécie possui características morfofisiológicas no seu epidídimo mais propícias à reprodução durante os meses secos na Mata Atlântica do Litoral Sul de Pernambuco.
Agradecimentos. A Coordenação de Aperfeiçoamento de Pessoal de Nível Superior (CAPES) pela bolsa de mestrado disponibilizada ao primeiro autor.

\section{REFERÊNCIAS}

Aguilera-Merlo, C.; Muñoz, E.; Dominguez, S.; Scardapane, L. \& PIEzzI, R. 2005. Epididymis of viscacha (Lagostomus maximus maximus): Morphological changes during the annual reproductive cycle. Anatomical Record 282:83-92.

Altringham, J. D. 1996. Bats. Biology and behavior. New York, Oxford University Press. 262p.

Artoni, S. M. B.; Manoel, H. L.; Lamano; Carvalho, T. L. L.; Orsi, A. M. \& Stefanini, M. A. 1999. Avaliação morfométrica da área do epidídimo e dos dúctulos eferentes e duetos epididimários da codorna doméstica, no decorrer do ano. Brazilian Journal of Veterinary Research and Animal Science 36(6):283-289.

Beguelini, M. R.; Góes, R. M.; Rahal, P.; Morielle-Versute, E. \& TABOGA, S. R. 2015. Impact of the Processes of Total Testicular Regression and Recrudescence on the Epididymal Physiology of the Bat Myotis nigricans (Chiroptera: Vespertilionidae). PLOS ONE 10:e 0128484 .

Beguelini, M. R.; Góes, R. M.; Taboga, S. R. \& Morielle-Versute, E. 2013c. Two periods of total testicular regression are peculiar events of the annual reproductive cycle of the black Myotis bat, Myotis nigricans (Chiroptera: Vespertilionidae). Reproduction, Fertility and Development 26:834-846.

Beguelini, M. R.; Puga, C. C. I.; Martins, F. F.; Betoli, A. H. S.; Taboga, S. R. \& Morielle-Versute, E. 2013a. Morphological Variation of Primary Reproductive Structures in Males of Five Families of Neotropical Bats. The Anatomical Record 296:156-167.

Beguelini, M. R.; Puga, C. C. I., Martins, F. F.; Negrin, A. C.; Christante, C. M.; Vilamaior, P. S. L.; Taboga, S. R. \& Morielle-Versute, E. 2012. Applications of Transmission Electron Microscopy to evaluate the diversity of the male reproductive system of Neotropical bats. In: MÉndeZ-VILAS, A. org. Current microscopy contributions to advances in science and technology. Badajoz, Formatex Research Center, p. 259-269.

Beguelini, M. R.; Puga, C. C. I.; Taboga, S. R. \& Morielle-Versute, E. 2013b. Annual reproductive cycle of males of the flat-faced fruit-eating bat, Artibeus planirostris (Chiroptera: Phyllostomidae). General and Comparative Endocrinology 185:80-89.

Beguelini, M. R.; Sergio, B. F. S.; Leme, F. L. J.; Taboga, S. R. \& Morielle-Versute, E. 2010. Morphological and morphometric characteristics of the epididymis in the Neotropical bats Eumops glaucinus and Molossus molossus (Chiroptera: Molossidae). Chiroptera Neotropical 16(2):769-779.

Behmer, O. A.; Tolosa, E. M. C. \& Freitas Neto, A. G. 1976. Manual de Técnicas para Histologia Normal e Patológica. São Paulo, EDART/ EDUSP. 331p.

Budras, K. D. \& Sauer, T. 1975. Morphology of the epididymis of the cock (Gallus domesticus) and its effect uppon the steroid sex hormone synthesis. Ontogenesis, morphology, and distribution of the epididymis. Anatomy and Embryology 148(2):175-196.

Calvo, A.; Bustos-Obregon, E. \& Pastor, L. M. 1997. Morphological and histochemical changes in the epididymis of hamsters (Mesocricetus auratus) subjected to short photoperiod. Journal of Anatomy 191:7788.

Calvo, A; Pastor, L. M.; Martínez, E.; Vázquez, J. M. \& Roca, J. 1999. Agerelated changes in the hamster epididymis. The Anatomical Record 256:335-346.

Campolina-Silva, G. H.; Hess, R. A. \& Oliveira, C. A. 2018. Seasonal variation of cell proliferation and apoptosis in the efferent ductules and epididymis of the Neotropical bat Artibeus lituratus (Chiroptera, Phyllostomidae). General and Comparative Endocrinology 273:3-10.

Castro, M. M.; Gonçalves, W. G.; Teixeira, S. A. M. V.; Queiroz Fialho, M. C.; Santos, F. C.; Oliveira, J. M.; Serrão, J. E. \& MachadoNEVES, M. 2017. Ultrastructure and morphometric features of epididymal epithelium in Desmodus rotundus. Micron 102:35-43. 
Cervantes, M. I.; Arenas-Rios, E.; León-Galván, M. A.; LóPEZ-Wilchis, R.; Ambriz, D. \& Rosado, A. 2008. Spermatozoa Epididymal Maturation in the Mexican Big-Eared Bat (Corynorhinus mexicanus). Systems Biology in Reproductive Medicine 54:196-204.

Cooper, T. 1999. Epididymis. In: Knobil, E. \& Neill, D. J. eds. Encyclopedia of reproduction, v.2. San Diego, Academic Press, p. 1-17.

Crichton, E. G. \& Krutzsch, P. H. 2000. Reproductive Biology of Bats. London, Academic Press. 510p.

EnCARnAÇÃo, J. A.; DietZ, M. \& KiERdorf, U. 2003. Reproductive condition and activity pattern of male Daubenton's bats (Myotis daubentonii) in the summer habitat. Mammalian Biology 69:163-172.

FABIÁN, M. F. \& MARQUES, R. V. 1989. Contribuição ao conhecimento da biologia reprodutiva de Molossus molossus (Pallas, 1766) (Chiroptera, Molossidae). Revista Brasileira de Zoologia 6(4):603-610.

Fleming, T. H.; HoOPER, E. T. \& WILSON, D. E. 1972. Three central American bat communities: structure, reproductive cycles and movement patterns. Ecology 53:555-569.

GuSTAFSON, A. W. 1979. Male reproductive patterns in hibernating bats. Journal of Reproduction and Fertility 56:317-331.

HinTON, B. 1990. The testicular and epididymal luminal amino acid micro environment in the rat. Journal of Andrology 11:498-505.

Hosken D. J.; Blackberry, M. A.; Stewart, T. B. \& Stuck, A. F. 1998. The male reproductive cycle of three species of Australian Vespertilionid bat. Journal of Zoology 245(3):261-270.

LeÓN-Galván, M. A.; LóPeZ-Wilchis, R.; HeRnÁndez-PÉReZ, O.; ArenasRíos, E. \& Rosado, A. 2005. Male reproductive cycle of Mexican big-eared bats, Corynorhinus mexicanus (Chiroptera: Vespertilionidae). The Southwestern Naturalist 50(4):453-460.

Lima Junior, N. B.; Arandas, M. J. G.; Marinho, K. S. N.; Aguiar Júnior, F. C. A.; Pontes, A. R. M. \& Santos, K. R. P. 2014. Histomorfometria testicular do morcego Phyllostomus discolor (Chiroptera: Phyllostomidae) em áreas de Mata Atlântica de Pernambuco. Brazilian Journal of Veterinary Researchand Animal Science 51(3):263-270.

Lima Junior, N. B.; Arandas, M. J. G.; Silva, F. R.; Antonio, E. A.; Aguiar Júnior, F. C. A.; Teixeira, A. A. C.; Garcia, J. E. \& Santos, K. R. P. 2018a. Analysis of the reproductive stage and ovarian histomorphometry of Dermanura cinerea (Chiroptera: Phyllostomidae) in an Atlantic Forest fragment of Pernambuco, northeastern Brazil. Pesquisa Veterinária Brasileira 38(1):167-174.

Lima Junior, N. B.; Arandas, M. J. G.; Silva, F. R.; Silva, D. V.; Teixeira, A. A. C.; Aguiar Júnior, F. C. A.; Garcia, J. E. \& Santos, K. R. P. 2018b. Estágio reprodutivo, histologia e morfometria sazonal do testículo de Dermanura cinerea (Chiroptera: Phyllostomidae) em fragmento de Mata Atlântica no Litoral Sul de Pernambuco, Brasil. Iheringia, Série Zoologia 108:e2018038.

Morais, D. B.; Paula, T. A. R.; Barros, M. S.; Balarini, M. K.; Freitas, M. B. D. \& MATTA, S. L. P. 2013. Stages and duration of the seminiferous epithelium cycle in the bat Sturnira lilium. Journal of Anatomy 222:372-379.

Neuweiler, G. 2000. The biology of bats. Oxford, Oxford University Press. 310p.

Oliveira, R. L.; Campolina-Silva, G. H.; Nogueira, J. C.; Mahecha, G. A. B. \& Oliveira, C. A. 2013. Differential expression and seasonal variation on aquaporins 1 and 9 in the male genital system of big fruit-eating bat Artibeus lituratus. General and Comparative Endocrinology 186:116-125.

Pastor-Soler, N. M.; Jane, S.; Sharpe, R.; Hill, E.; Van, H. A.; Brown, D. \& Breton, S. 2010. Aquaporin 9 expression in the developing rat epididymis is modulated by steroid hormones. Reproduction 139(3):613-621.

Redondo, R. A. F.; Brina, L. P. S.; Silva, R. F.; Ditch-Field, A. D. \& Santos, F. R. 2008. Molecular systematics of the genus Artibeus (Chiroptera: Phyllostomidae). Molecular Phylogenetics and Evolution 49:44-58.

Reis, N. R.; Peracchi, A. L.; Pedro, W. A. \& Lima, I. P. eds. 2011. Mamíferos do Brasil. 2ed. Londrina, Editora da Universidade Estadual de Londrina. 439p.

RIEDER, N. \& SCHMIDT, K. 1987. Morphologische Arbeitsmethoden in der Biologie. Weinheim, Wiley-VCH, Gesellsch.mbH. 232p.

Robaire, B. \& Hinton, B. T. 2015. The Epididymis. In: Plant, T. M. \& ZELEZNICK, A. J. Knobil \& Neill's Physiology of Reproduction. 4ed. Academic Press, p. 691-771.

Rocha P. A.; Mikalauskas J. S.; Gouveia S. F.; Silveira, V .V. B. \& Peracchi A. L. 2010. Morcegos (Mammalia, Chiroptera) capturados no Campus da Universidade Federal de Sergipe, com oito novos registros para o estado. Biota Neotropica 10(3):183-188.

Rochel, S. S.; Bruni-Cardoso, A.; Taboga, S. R.; Vilamaior, P. S. L. \& GóEs, R. M. 2007. Lobe identity in the Mongolian gerbil prostatic complex: a new rodent model for prostate study. The Anatomical Record 290:1233-1247.

Scultori, C.; Dias, D. \& Peracchi, A. L. 2009. Mammalia, Chiroptera, Phyllostomidae, Artibeus cinereus: First record in the state of Paraná, Southern Brazil. Check List Journal of Species Lists and Distribution 5(2):325-329.

Serre, V. \& Robaire, B. 1998. Segment-specific morphological changes in aging Brown Norway rat epididymis. Biology of Reproduction 58:497-513.

Solari, S.; Hoofer, S. R.; Larsen, P. A.; Brown, A. D.; Bull, R. J.; Guerrero, J. A.; Ortega, J.; Carrera, J. P.; Bradley, R. D. \& BAKER, R. J. 2009. Operational criteria for genetically defined species: analysis of the diversification of the small fruit-eating bats, Dermanura (Phyllostomidae: Stenodermatinae). Acta Chiropterologica 11(2):279-288.

Souza, A. C. F.; Santos, F. C.; Bastos, D. S. S.; Sertorio, M. N.; Teixeira, J. P. G.; Fernandes, K. M.; Machado-Neves, M. 2018. Reproductive functions in Desmodus rotundus: A comparison between seasons in a morphological context. PLoS ONE 13(10):e0205023.

Straube, F. C. \& Bianconi, G. V. 2002. Sobre a grandeza e a unidade utilizada para estimar esforço de captura comutilização de redes-deneblina. Chiroptera Neotropical 8:1-2.

TADDEI, V. A. 1980. Biologia reprodutiva de Chiroptera: Perspectivas e problemas. Inter-Facies Escritos e Documentos 6:1-18.

Tobón, A. R.; Arenas-Ríos, E. \& León-Galvan, M. A. 2010. El almacenamiento prolongado de espermatozoides en el epidídimo, adaptación que permite sincronizar el periodo de apareamiento sen murciélagos. ContactoS 78:58-64.

TURNER, T. T. 1991. Spermatozoa are exposed to a complex microenvironment as they traverse the epididymis. Annals of the New York Academy of Sciences 637:364- 383.

Wilson, D. E. 1973. Reproduction in neotropical bats. Periodicum Biologorum 75:214-217.

WiLson, D. E. 1979. Reproductive patterns. In: BAKER, R. J.; Jones, J. K. \& CARTER, D. C. ed. Biology of Bats of the New World - Family Phyllostomidae. Special Publications Museum. Lubbock, Texas Tech University, p. 317-318.

ZoRTÉA, M. 2003. Reproductive patterns and feeding habits of three nectarivorous bats (Phyllostomidae: Glossophaginae) from the Brazilian Cerrado. Brazilian Journal of Biology 63(1):159-168. 